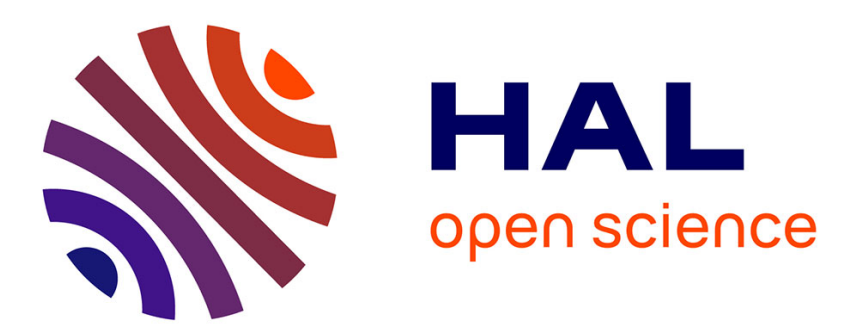

\title{
Dynamical simulation of collision-induced dissociation of pyrene dimer cation
}

\author{
Linjie Zheng, Sébastien Zamith, Mathias Rapacioli
}

\section{To cite this version:}

Linjie Zheng, Sébastien Zamith, Mathias Rapacioli. Dynamical simulation of collision-induced dissociation of pyrene dimer cation. Theoretical Chemistry Accounts: Theory, Computation, and Modeling, 2021, 140 (2), 10.1007/s00214-020-02716-7 . hal-03127909

\section{HAL Id: hal-03127909 \\ https://hal.science/hal-03127909}

Submitted on 1 Feb 2021

HAL is a multi-disciplinary open access archive for the deposit and dissemination of scientific research documents, whether they are published or not. The documents may come from teaching and research institutions in France or abroad, or from public or private research centers.
L'archive ouverte pluridisciplinaire HAL, est destinée au dépôt et à la diffusion de documents scientifiques de niveau recherche, publiés ou non, émanant des établissements d'enseignement et de recherche français ou étrangers, des laboratoires publics ou privés. 


\title{
Dynamical Simulation of Collision Induced Dissociation of Pyrene Dimer Cation
}

\author{
Linjie Zheng · Sébastien Zamith • Mathias Rapacioli
}

\begin{abstract}
We report a theoretical investigation of the collision induced dissociation of pyrene dimer cation, as recently investigated in the experimental work by Zamith et al. (J. Chem. Phys. 153, 054311 (2020)). Molecular dynamics simulations using potential energies and forces computed at the self-consistent charge density functional based tight binding level were conducted for different collision energies between 2.5 and $30 \mathrm{eV}$. It appears that most of the dissociation occurs on a short timescale (less than $3 \mathrm{ps}$ ). The dynamical simulations allow to visualise the dissociation processes. At low collision energies, the dissociation cross section increases with collision energies whereas it remains almost constant for collision energies greater than 10$15 \mathrm{eV}$. The analysis of the kinetic energy partition is used to get insights into the collision/dissociation processes at the atomic scale. The simulated time of flight mass spectra of parent and dissociation products are obtained from the combination of molecular dynamics simulations and phase space theory to address the short and long timescales dissociation, respectively. The agreement between the simulated and measured mass spectra suggests that the main processes are captured by this approach.
\end{abstract}

L. Zheng · M. Rapacioli (四)

Laboratoire de Chimie et Physique Quantique (LCPQ/IRSAMC), UMR5626, Université de Toulouse (UPS) and CNRS, 118 Route de Narbonne, F-31062 Toulouse, France

E-mail: mathias.rapacioli@irsamc.ups-tlse.fr

S. Zamith

Laboratoire Collisions Agrégats Réactivité (LCAR/IRSAMC), UMR5589, Université de Toulouse (UPS) and CNRS, 118 Route de Narbonne, F-31062 Toulouse, France
Keywords Molecular Dynamics · SCC-DFTB ·

Collision Induced Dissociation · Pyrene Dimer Cation · Polycyclic Aromatic Hydrocarbon Clusters

\section{Introduction}

Clusters of polycyclic aromatic hydrocarbons (PAH) are relevant systems in several scientific fields, including astrophysics and combustion sciences. The role of PAH clusters in combustion processes is still under debate, in particular as they might or not be intermediate systems in the soot particles growth [1-10]. In astrophysics, PAHs have been proposed in the mid-eighties to explain a series of infrared emission bands ubiquitous in the Universe $[11,12]$. The broadening of these bands in regions protected from the star's UV flux suggests the following scenario: PAHs are trapped in clusters in UV-protected regions and photo-evaporated by star's UV photons in the so-called photodissociated regions [13-15].

The investigation of PAH clusters has been performed from both the experimental and theoretical sides. From the experimental side, many studies focused on the investigation of structural properties of these clusters at the most stable geometrical configurations [5, 7, 16-19]. Their energetic properties such as ionisation potentials have been recorded [20] as well as their spectral properties [21-24]. The stability of PAH clusters has also been investigated from experiments following the evaporation of these clusters after absorption of a photon or a collision process [25-31]. The range of collision energies considered experimentally is quite large, ranging from $\mathrm{eV}$ to high energy collision at a few keV. Low energy collision experiments allow for the derivation of dissociation energies [32] whereas the PAHs oligomerization within the cluster induced by 
high energy collisions [31] or photoabsorption [33-35] suggest the possible role of clusters in the interstellar PAHs growth process [36].

From the theoretical side, the size of the systems limits the use of $a b$ initio wave function methods to the investigation of properties of the smallest clusters, namely dimers [18, 37-39], whereas larger clusters can be addressed either at the DFT level or with more semiempirical schemes [2, 5, 18, 23, 38, 40-57]. Many of these studies, focused on structural properties, evidence a stacking growth process in agreement with experimental results. IR properties were also reported at the DFT level [45]. Most of the theoretical studies involve neutral clusters, mostly due to the fact that treating charge resonance process in ions is a challenging task for DFT based methods [58]. One should however mention recent studies computing ionisation potentials [20] as well as structural [59] and spectral (electronic [60] and vibrational [61]) properties of cations, performed with an original model combining density functional based tight binding (DFTB) [62-65] with a configuration interaction scheme[66].

With respect to these studies, very few is known about the dynamical aspects of PAH clusters carrying internal energy. High energy collisions of PAH clusters with energetic ions have been simulated by Gatchel et al. $[67,68]$ at the semi-empirical and DFTB levels. Recently experiments at lower collision energies were performed [69]. These were analysed by treating statistically the dissociation after collision energy deposition. Namely, the dissociation rate of pyrene clusters has been computed using phase space theory (PST)[32]. A fair agreement with experimental results was obtained concerning the collision energy dependence of the dissociation cross section. However, the employed model failed at reproducing in details the shape of the peaks in the time of flight (TOF) spectra. In the present work, we aim at extending the description of such low energy collision processes (less than several tens of eV) combining a dynamical simulation to describe the fast processes in addition to the statistical theory to address dissociation at longer timescales. With this approach, (i) we show good agreement between simulated and experimental mass spectra, thus validating the model, (ii) we derive dissociation cross sections as a function of the collision energy, (iii) we discuss the kinetic energy partition between dissociative and non-dissociative modes and (iv) the energy transfer efficiency between intra and intermolecular modes.

The paper is organized as follows: In Section 2, the computational methods are described. In Section 3, we present the comparison of the simulated TOF mass spectra with the experimental ones and analyse and discuss the molecular dynamics results. Conclusions are drawn in Section 4.

\section{Computational Approach}

\subsection{Dynamics simulations of the collision process}

The dynamics simulations of the collision process were performed with a QM/MM scheme where the Argon is treated as a polarisable MM particle interacting with the pyrene dimer cation $\mathrm{Py}_{2}^{+}$, the latter being treated at the DFTB level, an approximated DFT scheme whose computational efficiency relies on the use of parameterized integrals $[62-64,70,71]$. The details about this QM/MM scheme can be found in the original paper [72]. In this work, we used the second-order version of DFTB, SCC (self-consistent-charge)-DFTB [64], with the matsci-0-3 parameters [73]. To improve the intermolecular interactions, Mulliken charges were replaced by the Charge Model 3 (CM3) charge definition [42, 74] and empirical terms were used to describe dispersion interactions [42]. A Fermi distribution, using a temperature of $2000 \mathrm{~K}$, was applied to determine the molecular orbitals occupations. This is done in order to avoid oscillation problems during the search for a self-consistent solution. This oscillation problem often appears for dissociated or close to dissociation systems. Furthermore, the Fermi distribution allows to recover the continuity in energy and gradients in the case of level crossing [75]. We also mention that, in order to keep a low computational cost, no correction has been used to improve the DFTB charge resonance description. However, this charge delocalization issue has been specifically addressed in the case of $\mathrm{PAH}$ cation dissociation and it was shown to have a minor effect on the final computed mass spectra [76]. We also mention that the collision energy is in principle high enough to have electronic excitation in the system, which is taken into account at a crude level by the use of a Fermi temperature. Finally, nuclear quantum effects are not taken into account. Although this may affect the results at very low collision energies, the effect is expected to be small for the experimental collision energy of $17.5 \mathrm{eV}$. Although all these limits should be kept in mind, we would like to emphasize that, recently, the dissociation of $\mathrm{PAH}$ molecules has been simulated and a good agreement with experimental results was obtained despite similar crude approximations, namely neglect of non-adiabatic and nuclear quantum effects, improper treatement of charge delocalization and use of a Fermi temperature [76-78].

Concerning the MD preparation, starting from the optimized $\mathrm{Py}_{2}^{+}$geometry [59], a preliminary thermal- 
ization run of 200 fs at $25 \mathrm{~K}$ (maintained by a NoséHoover chain thermostat $[79,80])$ is performed. Then, the Argon atom projectile is introduced in the simulation with a velocity determined to reproduce a given collision energy. The target dimer cation was positioned at the origin of the simulation referential and randomly rotated to allow all possible impact points on the dimer. The Argon atom is initially positioned at $\mathrm{x}=10, \mathrm{y}=b$ and $\mathrm{z}=0 \AA$, with $b$ being the impact parameter. At each center of mass collision energy $E_{\text {col }}$ from 2.5 to $30.0 \mathrm{eV}$, a series of 300 collision trajectories were conducted for each of the 13 values of $b$ evenly distributed between 0 and $6 \AA$. Trajectory calculations have been performed with a time step of 0.5 fs and a total duration of 3 ps. We have checked that for high collision energies (20 and $25 \mathrm{eV}$ ) a time step of $0.1 \mathrm{fs}$ does not change significantly our numerical results (see Table 1, Figures 1, 2 and 3 of the supplementary materials).

During the results collection, we extract, for each trajectory, the final snapshot and consider that the dimer is dissociated if the distance between the two monomers molecular mass centers is larger than $10 \AA$. The opacity $P\left(b, E_{c o l}\right)$, i.e. the dissociation probability as a function of impact parameter at a given collision energy is computed by averaging the results over the simulations corresponding to these conditions. The cross sections are then derived from the following formula:

$$
\begin{aligned}
\sigma\left(E_{c o l}\right) & =\int_{0}^{b_{\max }} 2 \pi P\left(b, E_{c o l}\right) b d b \\
& \simeq \sum_{i} \frac{P\left(b_{i}, E_{c o l}\right)+P\left(b_{i+1}, E_{c o l}\right)}{2} \pi\left(b_{i+1}^{2}-b_{i}^{2}\right)
\end{aligned}
$$

Mean values are computed using the same approach, followed by a division by $\pi b_{\max }^{2}$. When mean values are restricted to trajectories leading to dissociation (noted $-d$ ) or not (noted $-u d$ ), additional normalisation by the total number of dissociated or undissociated trajectories is also necessary. In the analysis, we will also discuss kinetic energy contributions, applying the fol- lowing decomposition of the total kinetic energy $E_{t o t}^{k}$ :

$$
\begin{aligned}
E_{t o t}^{k} & =E_{A r}^{k}+E_{t d}^{k}+E_{P y^{1}}^{k}+E_{P y^{2}}^{k}+E_{R e}^{k} \\
E_{A r}^{k} & =\frac{1}{2} m_{A r} \vec{v}_{A r}^{2} \\
E_{t d}^{k} & =\frac{1}{2} m_{P y_{2}} \vec{v}_{t}^{2}\left(P y_{2}\right) \\
E_{R e}^{k} & =\frac{1}{2} \frac{m_{P y^{1}} m_{P y^{2}}}{m_{P y^{1}}+m_{P y^{2}}}\left(\vec{v}_{t}\left(P y^{2}\right)-\vec{v}_{t}\left(P y^{1}\right)\right)^{2} \\
E_{P y^{n}}^{k} & =\frac{1}{2} \sum_{i=1}^{26} m_{i}^{n}\left(\vec{v}_{i}^{n}-\vec{v}_{t}\left(P y^{n}\right)\right)^{2} \\
E_{\text {intran }}^{k} & =\frac{1}{2} \sum_{i=1}^{26} m_{i}^{n}\left(\vec{v}_{i}^{n}-\vec{v}_{t}\left(P y^{n}\right)-\vec{v}_{i r}^{n}\right)^{2} \\
E_{\text {inter }}^{k} & =E_{\text {tot }}^{k}-E_{A r}^{k}-E_{t d}^{k}-E_{r}^{k}-E_{\text {intra }{ }^{1}}^{k}-E_{\text {intra }}^{k}
\end{aligned}
$$

In these equations and in the following, $P y_{2}$ refers to the pyrene dimer (possibly dissociated) whereas $P y^{1}$ and $P y^{2}$ refer to the first and second monomers, respectively. $E_{A r}^{k}$ refers to the kinetic energy of the Argon (with mass $m_{A r}$ and velocity $\vec{v}_{A r}$ ). $E_{t d}^{k}$ is the translation kinetic energy of the dimer (with mass $m_{P y_{2}}$ and velocity $\left.\vec{v}_{t}\left(P y_{2}\right)\right)$. $E_{R e}^{k}$ is the relative kinetic energy of the two pyrene monomers, computed from their masses of $m_{P y^{1}}=m_{P y^{2}}$ and monomer global translation velocities $\vec{v}_{t}\left(P y^{n=1,2}\right)$. $E_{P y^{n}}^{k}$ is the rovibrational kinetic energy of the monomer $n$ computed from the masses and velocities of its atoms $\left(m_{i}^{n}\right.$ and $\left.\vec{v}_{i}^{n}\right)$.

$E_{\text {intra }}^{k}$ is the intramolecular vibrational kinetic energy of monomer $n$ obtained after removing the contributions associated to the monomer translation and rotation modes. $\vec{v}_{i r}^{n}$ is the velocity of atom $i$ associated to the monomer global rotation. Finally, $E_{r}^{k}$ refers to the rotation kinetic energy of the dimer and $E_{\text {inter }}^{k}$ is the dimer intermolecular kinetic energy.

From the endpoint of the simulation, we can also compute the total energy transferred towards internal rovibrational modes of the pyrene dimer as:

$\Delta E_{i n t}^{P y_{2}}=E_{A r}^{k, 0}-E_{A r}^{k}-E_{t d}^{k}$

where $E_{A r}^{k, 0}$ is the initial Argon kinetic energy whereas $E_{A r}^{k}$ and $E_{t d}^{k}$ correspond to kinetic energies at the end of the MD simulation. In the case of dissociated dimers at the end of the simulations, we can also deduce the energy deposited in the rovibrational modes of the monomers as:

$\Delta E_{i n t}^{P y^{1}+P y^{2}}=E_{A r}^{k, 0}-E_{A r}^{k}-E_{t d}^{k}-E_{R e}^{k}$

All the calculations in the present work were carried out with the deMonNano code [81]. 
2.2 Simulation of the experimental time of flight mass spectra

Before describing how the experimental time of flight mass spectra (TOFMS) are simulated, we briefly describe below how these are obtained. Pyrene cationic clusters are produced in a gas aggregation source and thermalized at a temperature of $25 \mathrm{~K}$. Clusters are then mass-selected with a chosen kinetic energy. They collide with Argon atoms in a collision cell. The collision products are then analysed by time of flight mass spectrometry. Further details about the experiment can be found elsewhere [32, 69, 82, 83].

These experimental TOFMS are reproduced by simulating the ion trajectories through the experimental setup in the presence of the electric fields. These are calculated by solving numerically the Laplace equation. Equations of motion are integrated using the 4th order Runge-Kutta method with adaptive step size. The occurrence of collision or dissociation is decided at each time step of the ion trajectory based on the collision and dissociation probabilities. In our previous study [69] we treated the energy transfer upon collision by using the Line of Center model (LOC) [84]. In this model, the transferred energy is the kinetic energy along the line of centers. Evaporation rates were then estimated using PST. In this approach, we therefore only consider statistical dissociation to be possible after energy deposition in the cluster by collision. Although this approach, that will be referred to as PST in the following, has been proved to be able to satisfactorily reproduce CID cross section experiments, it fails to reproduce in details the shape and position of the fragment peaks in the TOFMS, as will be shown shortly.

In order to better reproduce the position and peak shapes, we have used the output of the MD simulations presented in Section 2.1 to treat the collisions in the ion trajectories. At each time step the probability for a collision is evaluated. If a collision occurs, we then randomly pick one of the MD calculations (with proper weighting of the $b$ values). Two cases have then to be considered. First, if the dissociation occurred during the picked MD calculation, then we use the MD final velocities of the fragments to further calculate the ion trajectories. On the other hand, if the pyrene dimer is still intact at the end of the picked MD calculation, then we update the dimer velocity and use the collision energy transfer $\Delta E_{i n t}^{P y_{2}}$ deduced from the MD calculation to increase the internal energy of the cluster. The dissociation rate resulting from this new internal energy is then evaluated using PST. In the latter case, if dissociation occurs, the relative velocities of the fragment are evaluated using the PST outcome. This approach will be referred to as the MD+PST scheme.

We emphasize here that, due to the short time scale of the MD calculations (3 ps), only direct dissociation can be captured by the MD simulations. Therefore, one has to evaluate the probability of dissociation at longer time scales after the energy deposition by collision. This is done here by considering that at longer time scales, dissociation occurs statistically and is treated by using PST.

In the following, we will refer to dissociation at short, experimental or infinite timescales. The first two ones correspond to dissociation occurring during the MD simulation only or with the MD+PST model. The dissociation at infinite time accounts for all MD trajectories where the amount of energy transferred to the internal dimer rovibrational modes $\Delta E_{i n t}^{P y_{2}}$ is larger than the dissociation energy of $1.08 \mathrm{eV}$ (value from references $[59,69])$. It can be regarded as the dissociation occurring after an infinite time neglecting any cooling processes, such as thermal collisions or photon emissions.

\section{Results and Discussion}

\subsection{Mass spectrum comparison}

An example of TOFMS is given in Figure 1. The Figure 1 (a) is centered around the intact parent mass $\left(\mathrm{Py}_{2}^{+}\right)$whereas in (b) is displayed the region around the fragment peak $\left(\mathrm{Py}^{+}\right)$.

In Figure 1(a) are displayed three curves corresponding to the experimental one and the results of the two simulations (PST and MD+PST) for the parent ion. One can see that the peak shape and position are properly reproduced using the simulations, therefore the essential of the ion propagation is captured by the simulations. Although some of the detected parent ions have undergone a collision without dissociation, no difference is seen in the parent peak since the collision rate is kept very small.

In Figure 1(b), the experimental result is compared to the PST and MD+PST simulations. Clearly, the PST based simulation fails to reproduce both the position and the shape of the peak. On the other hand, a much better agreement is found when using the output of the MD+PST simulations. This agreement is a good indication that this scheme captures the essence of the pyrene dimer cation dissociation induced by Argon collisions at this collision energy. Actually, in this scheme, the largest contribution to the TOF spectra results from dimers dissociating on short timescales, i.e. during the 

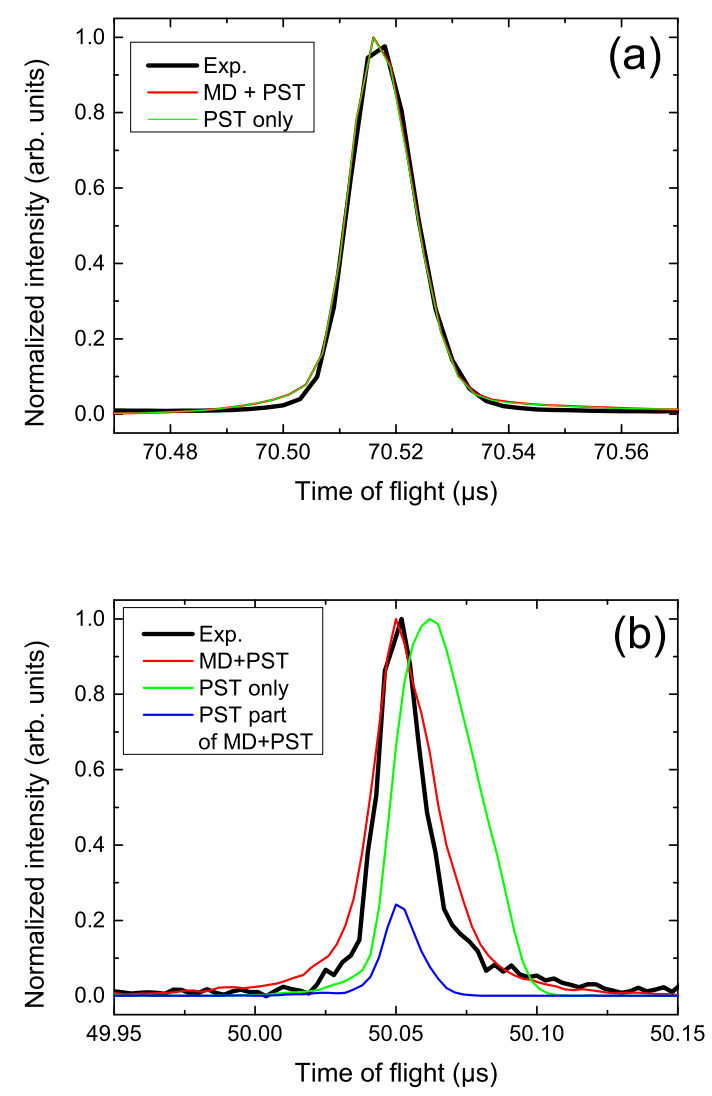

Fig. 1 Normalized time of flight mass spectra of (a) the parent pyrene dimer cation and (b) the pyrene fragment $\mathrm{Py}^{+}$ resulting from the collision of $\mathrm{Py}_{2}^{+}$with Argon at a center of mass collision energy of $17.5 \mathrm{eV}$. The black line is for the experimental result whereas red and green curves are the MD+PST and PST model results. The blue curve is the PST subcontribution of the MD+PST model.

MD simulation. The remaining contribution, i.e. resulting from dimers dissociating at longer timescales and computed from the second step PST calculation, is minor and represented in blue in Figure 1 (b).

\subsection{Molecular dynamics analysis}

\subsubsection{Description of selected trajectories}

A first qualitative description of the collision processes can be obtained from the analysis of some arbitrarily selected MD trajectories. Figure 2 (top and middle) reports some snapshots extracted from two trajectories with the same collision energy $(17.5 \mathrm{eV})$ and impact parameter $(3.5 \AA)$. Only the first one leads to the $\mathrm{Py}_{2}^{+}$ dissociation. Figures $2-1 / 1$ * represent the system after its preliminary thermalization, when the Argon atom introduced in the simulation with its initial velocity. Figures $2-2 / 2^{*}$ and $3 / 3^{*}$ represent the beginning and end of the collision. From these points, the two trajectories show different behaviors. For the first trajectory, snapshot 5 corresponds to the step where the two pyrene monomers start to go away from each other. After this, the intermolecular distance continues to increase further in snapshot 6 . For the second trajectory, Figures $2-5^{*}$ and $6^{*}$ correspond to the middle and ending snapshots of the simulation, respectively. The snapshots $4^{*}, 5^{*}$ and $6^{*}$ show the process of energy redistribution within the clusters. In particular, the soft modes associated to global deformation of the molecular planes appear to be excited. From these two particular cases, it can be seen that the evolution of the trajectory either toward a dissociation or a redistribution of the transferred energy strongly depends on the process of energy transfer during the collision. In the first case, the Argon atom is pushing the two monomers far away from each other, i.e. the transferred energy is mostly localised in an intermolecular dissociative mode. On the opposite, in the second trajectory, the collision mostly involves an intramolecular soft vibrational mode. The transferred energy is then redistributed over all the other modes. The statistical distribution of this energy is then hardly favorable to the dissociation due to the large number of intramolecular modes (72 per pyrene) with respect to the 6 intermolecular modes, only 3 of them ( 1 breathing and 2 parallel displaced modes) being dissociative modes.

The amount of transferred energy is also a major ingredient for the fate of the cluster. Depending on the details of the collision such as impact parameter or cluster orientation, very different amounts of energy can be transferred. This is illustrated in Figure 3 where the distribution of transferred energy $\Delta E_{\text {int }}^{P y_{2}}$ restricted to trajectories that would dissociate after infinite time, is plotted for simulations at the experimental collision energy of $17.5 \mathrm{eV}$. This distribution could hardly be guessed without a dynamical description of the collision at the atomic level. Indeed, a simpler model such as the LOC model (used in the pure PST approach) would lead to a constant distribution between the binding energy and the maximum collision energy as shown in Figure 3. In the distribution resulting from MD simulations, lower transferred energies are favored with respect to the distribution extracted from the LOC model. All these effects are intrinsically taken into account in the MD simulations on the opposite to the pure PST model, explaining the better agreement of the MD+PST scheme with the experimental results.

Finally, we note that the pyrene monomers remained intact (no fragmentation) up to collision en- 

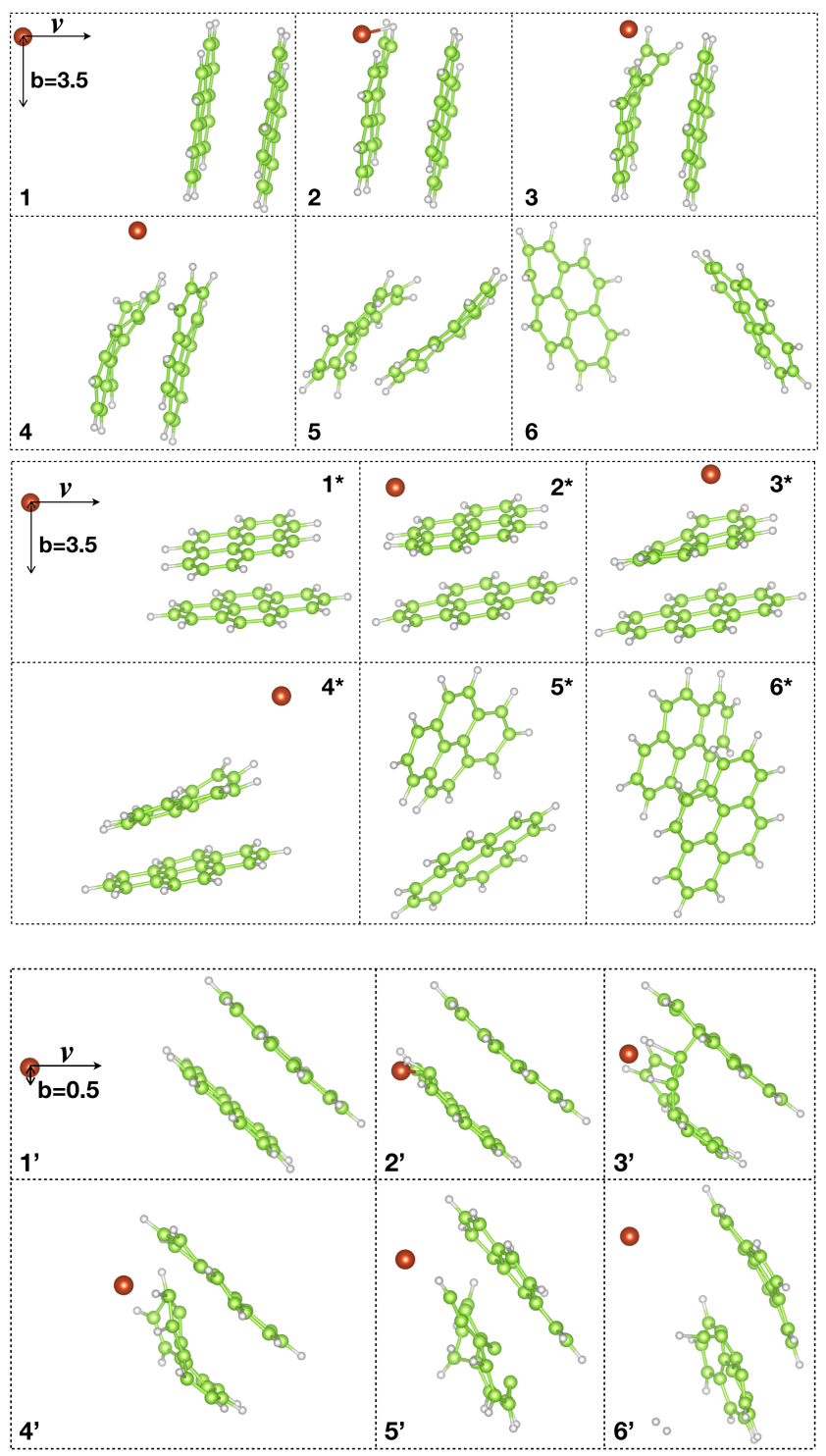

Fig. 2 Snapshots for three different molecular dynamics trajectories. Top and middle: trajectories with impact parameter of $3.5 \AA$ and a collision energy of $17.5 \mathrm{eV}$, leading to dissociation or non-dissociation (top and middle, respectively). Bottom: trajectory with impact parameter of $0.5 \AA$ and a collision energy of $27.5 \mathrm{eV}$ leading to intramolecular fragmentation.

ergies of $25 \mathrm{eV}$. The snapshots of a fragmentation trajectory at collision energy of $27.5 \mathrm{eV}$ are shown at the bottom of Figure 2. It can be seen that the pyrene molecule impacted by the Argon undergoes an opening of an aromatic cycle and the loss of two hydrogen atoms, leaving as a $\mathrm{H}_{2}$ molecule. As the study of monomer's fragmentation is beyond the scope of the present paper, we will focus on trajectories with collision energies below this fragmentation threshold energy in the following.

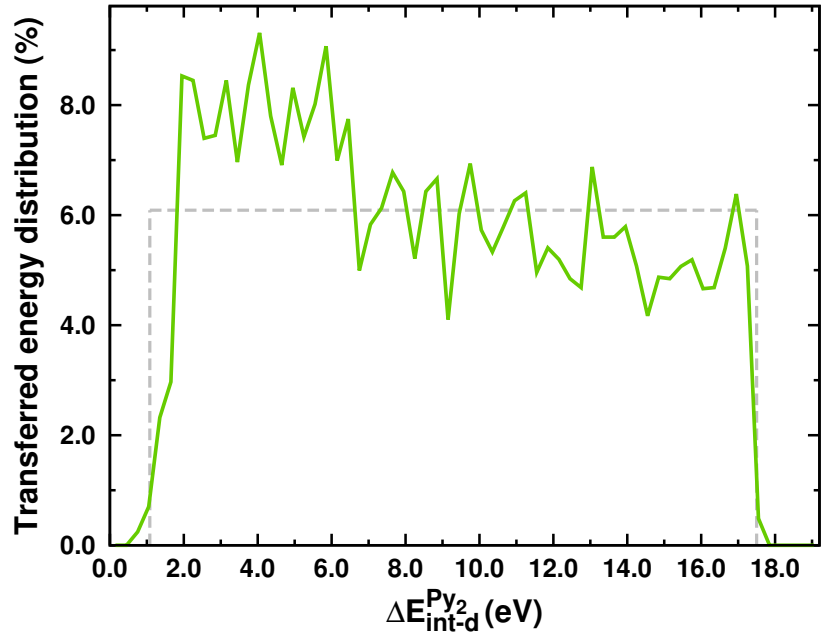

Fig. 3 Distribution of transferred energy in rovibrational modes $\Delta E_{i n t}^{P y_{2}}$ for trajectories leading to dissociation at the end of MD (center of mass collision energy of $17.5 \mathrm{eV}$ ). The dashed line shows the distribution of transferred energy used in the LOC model.

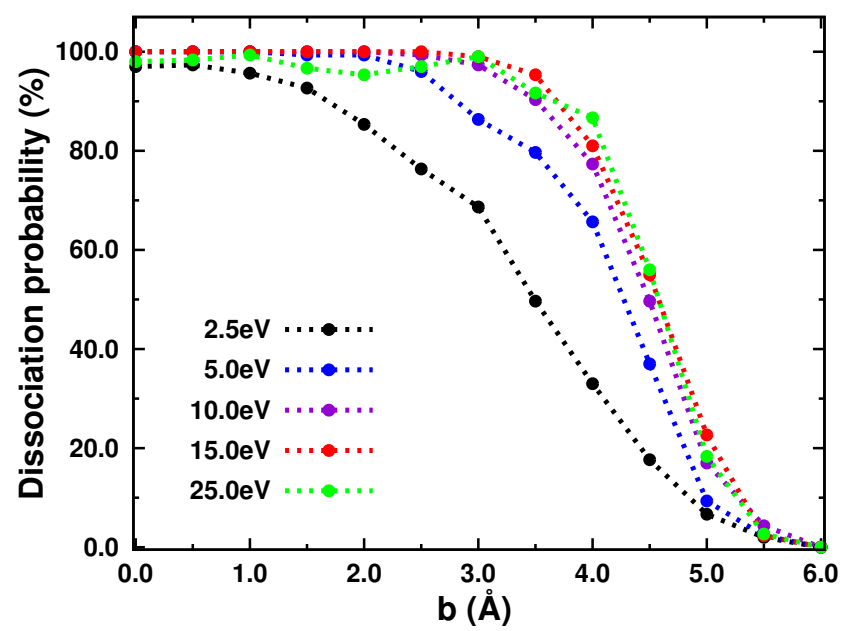

Fig. 4 Opacity curves as a function of the impact parameter $b$ for several selected center of mass collision energies.

\subsubsection{Dissociation cross section}

The opacity curves are presented in Figure 4 for various collision energies. At low impact parameters, the dissociation is very efficient even at low collision energy. At the lowest collision energy of $2.5 \mathrm{eV}$, the opacity curve presents a smooth decrease from 2 to $5 \AA$, whereas for collision energies larger than $10 \mathrm{eV}$, all curves are very similar. These high energy curves show high dissociation probability below $3.5 \AA$, reach $50 \%$ at about $4.5 \AA$ and drop to zero for values larger than $5.5 \AA$. These values can be compared to the van der Waals radius of Argon $(1.88 \AA)$ plus half of (i) the distance between the two molecules centers of masses $(3.04 \AA)$, (ii) the smallest $(6.82 \AA)$ or (iii) largest pyrene axes $(9.25 \AA)$ lead- 
ing to distances of $3.40,5.29$ and $6.50 \AA$, respectively. Below $3.40 \AA$, all trajectories involve a frontal impact of the Argon on the dimer carbonaceous system and almost all of them lead to dissociation. Unexpectedly, the opacity curve drops to zero at $5.5 \AA$ which is lower than the largest computed value of $6.5 \AA$. Interestingly, taking the largest distance between carbon atoms in pyrene $(7.0 \AA)$ instead of that between hydrogen atoms $(9.25 \AA)$ leads to a value of $5.4 \AA$ which is in line with the opacity curves. This suggests that the dissociation is efficient only if the carbonaceous skeleton area is impacted, the impact in the region of external hydrogen atoms resulting mostly in an intramolecular $\mathrm{C}-\mathrm{H}$ mode excitation at the expense of dissociative modes. As a conclusion, it seems that for energies larger than $10 \mathrm{eV}$, the opacity curves are similar as they are driven by simple geometric rules, in other words, if the dimer receives a direct impact of the Argon on the carbonaceous skeleton area, it will dissociate. Interestingly, this seems to be in agreement with previous works $[68,85]$ which also pointed out the efficient nuclear stopping power of carbon atoms in a very different context (higher energy collisions leading to knock-out process).

The blue curve in Figure 5 shows the MD dissociation cross sections of pyrene dimers obtained from the opacity curves following Eq. 1. It presents a steep increase for energies bellow $7.5 \mathrm{eV}$ before remaining almost constant around $65 \AA^{2}$ for collision energies greater than $10-15 \mathrm{eV}$. This is a direct consequence from the already discussed similarity of opacity curves for the high collision energies. The purple curve corresponds to dissociation at infinite timescales. Figure 5 also reports the cross sections computed from the MD+PST model. It can be seen that, for low collision energies, the MD and MD+PST cross sections are very close, indicating that most of the dissociations occur on the short timescales. On the opposite, at high collision energies, a non-negligible fraction of the dimers, which are not dissociated at the end of the MD simulation, carry enough energy to evaporate on the experimental timescales. At the experimental center of mass collision energy of $17.5 \mathrm{eV}$, the MD+PST cross section (about $70 \AA^{2}$ ) is slightly above the pure MD dissociation ratio, which indicates that the dissociation at long timescales represents a small fraction of the dissociated pyrene dimers as already seen from the TOF spectra analysis (see Figure 1).

We have also plotted in Figure 5 the model cross section that successfully reproduced the threshold collision induced dissociation experimental results [69]. This model cross section is obtained by considering that the collision energy transfer is given by the LOC model and

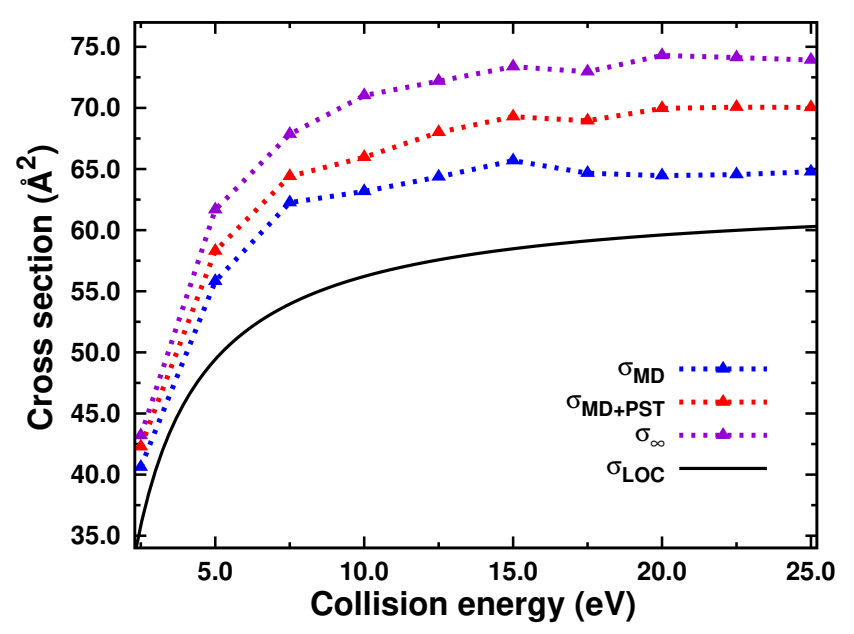

Fig. 5 Dissociation cross sections of $\mathrm{Py}_{2}^{+}$after collision with Argon as a function of center of mass collision energy for the short (MD), experimental (MD+PST) and infinite timescales. Cross sections resulting from the LOC model are also plotted.

the expression for the cross section is given by:

$\sigma_{L O C}\left(E_{c o l}\right)=\sigma_{0}\left(E_{c o l}-D\right) /\left(E_{c o l}\right)$.

where $D=1.08 \mathrm{eV}$ is the dissociation energy $[59,69]$ and $\sigma_{0}=63 \AA^{2}$ is a scaling factor usually thought as the geometrical cross section. This model cross section is usually further convolved with dissociation rates, collision energy distributions and internal energy distributions in order to be compared with experimental results. However, since here for the theoretical calculations there is no collision energy distribution, this curve could in principle be directly compared with the purple one in Figure 5, namely the cross section for infinite time. One can see that the MD, MD+PST results and the model cross section have similar collision energy dependence. The magnitude of the two cross sections is rather different at high collision energy, with about $60 \AA^{2}$ and $74 \AA^{2}$ for the model and infinite timescale cross sections respectively. Nevertheless, this difference is probably within the error bars of the experimental cross section measurement.

\subsubsection{Energy partition}

The mean value obtained for the transferred energy after removing the translation kinetic energy of the dimer, namely $\Delta E_{i n t}^{P y_{2}}$, is plotted in Figure 6 as a function of the collision energy. Although this quantity evolves almost linearly with the collision energy, the curves are different when one considers only the trajectories leading to dissociation or non-dissociation. For trajectories where the dimer does not dissociate, $\Delta E_{\text {int-ud }}^{P y_{2}}$ remains small for all collision energies below $20 \mathrm{eV}$ and shows 


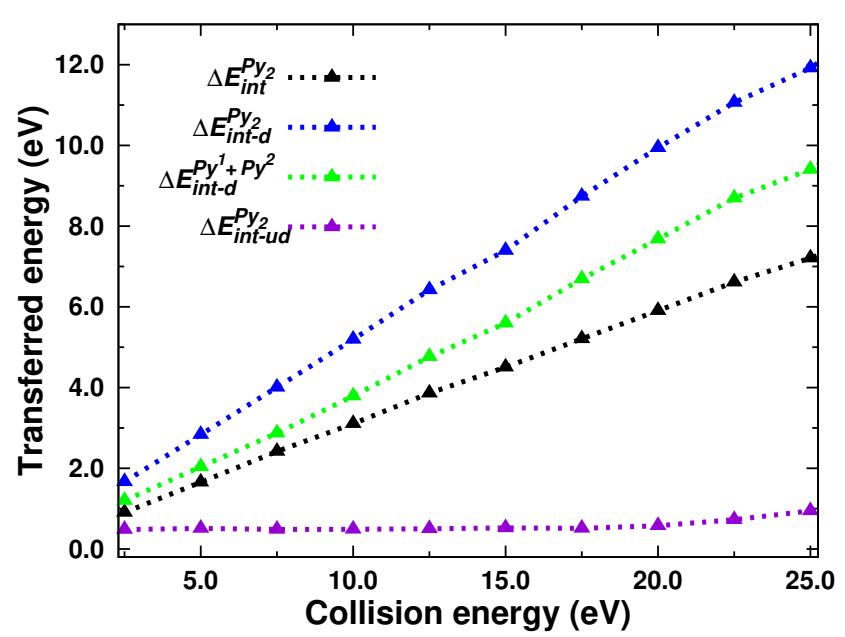

Fig. 6 Total transferred energy $\Delta E_{i n t}^{P y_{2}}$ to the rovibrational modes or restricted to the sole dissociated $\left(\Delta E_{i n t-d}^{P y_{2}}\right)$ or undissociated $\left(\Delta E_{i n t-u d}^{P y_{2}}\right)$ pyrene dimers as a function of collision energy. The transeferred energy to the monomers rovibrational modes for the dissociated dimers $\Delta E_{i n t-d}^{P y^{1}+P y^{2}}$ is also plotted.

a very slight increase for collision energies larger than $20 \mathrm{eV}$. For trajectories leading to dissociation, $E_{i n t-d}^{P y_{2}}$ grows almost linearly, but above $10-15 \mathrm{eV}$ most of the absorbed energy is actually used to heat the individual monomers (the green curve) whereas the energy given in the dissociative mode (difference between the blue and green curves) remains almost constant. We note that, despite the trends of the mean energy values derived from all simulations or restricted to the undissociated cases are interesting, their absolute values have small meaning as they depend on the arbitrarily chosen $b_{\max }$ value, i.e. increasing $b_{\max }$ would result in more undissociated trajectories with less and less energy transfer. On the opposite, absolute values of mean energies for the dissociation trajectories are relevant, as increasing the $b_{\max }$ value would not result in new dissociation trajectories.

It is also interesting to focus on the kinetic energy partition, in particular because its decomposition in sub-contributions (dissociative vs non-dissociative modes) is easier (see Eqs. 2) than that of the potential (and consequently total) energy. For each simulated collision energy, the values for the kinetic energy subcontributions (Eqs. 2) are averaged over all the trajectories and reported in Table 1 (Figure 1 in the supplementary material document). In Figure 7 are reported the ratios of the pyrene dimer translational kinetic energy $E_{t d}^{k}$, relative kinetic energy $E_{R e}^{k}$ and monomers rovibrational kinetic energies $E_{P y^{1}}^{k}+E_{P y^{2}}^{k}$ over the total pyrene dimer kinetic energy $E_{t o t}^{k}-E_{A r}^{k}$. It clearly appears that, whereas the contribution of the dimer translation ki-
Table 1 The kinetic energy partition after the collision of pyrene dimer with Argon at different collision energies $E_{c o l}$. All energies are in $\mathrm{eV}$.

\begin{tabular}{c|c|c|c|c|c}
$\boldsymbol{E}_{\boldsymbol{c o l}}$ & $\boldsymbol{E}_{\boldsymbol{t} \boldsymbol{d}}^{\boldsymbol{k}}$ & $\boldsymbol{E}_{\boldsymbol{A r}}^{\boldsymbol{k}}$ & $\boldsymbol{E}_{\boldsymbol{P} \boldsymbol{y}^{\mathbf{1}}}^{\boldsymbol{k}}$ & $\boldsymbol{E}_{\boldsymbol{P} \boldsymbol{y}^{\mathbf{2}}}^{\boldsymbol{k}}$ & $\boldsymbol{E}_{\boldsymbol{R} \boldsymbol{e}}^{\boldsymbol{k}}$ \\
\hline 2.5 & 0.17 & 1.67 & 0.25 & 0.25 & 0.19 \\
5.0 & 0.30 & 3.52 & 0.40 & 0.41 & 0.41 \\
7.5 & 0.42 & 5.38 & 0.55 & 0.56 & 0.64 \\
10.0 & 0.53 & 7.32 & 0.71 & 0.70 & 0.80 \\
12.5 & 0.65 & 9.20 & 0.89 & 0.88 & 0.95 \\
15.0 & 0.74 & 11.20 & 1.03 & 1.03 & 1.06 \\
17.5 & 0.82 & 13.16 & 1.16 & 1.22 & 1.18 \\
20.0 & 0.89 & 15.12 & 1.37 & 1.32 & 1.30 \\
22.5 & 0.96 & 17.09 & 1.52 & 1.51 & 1.36 \\
25.0 & 1.01 & 19.18 & 1.61 & 1.69 & 1.45
\end{tabular}

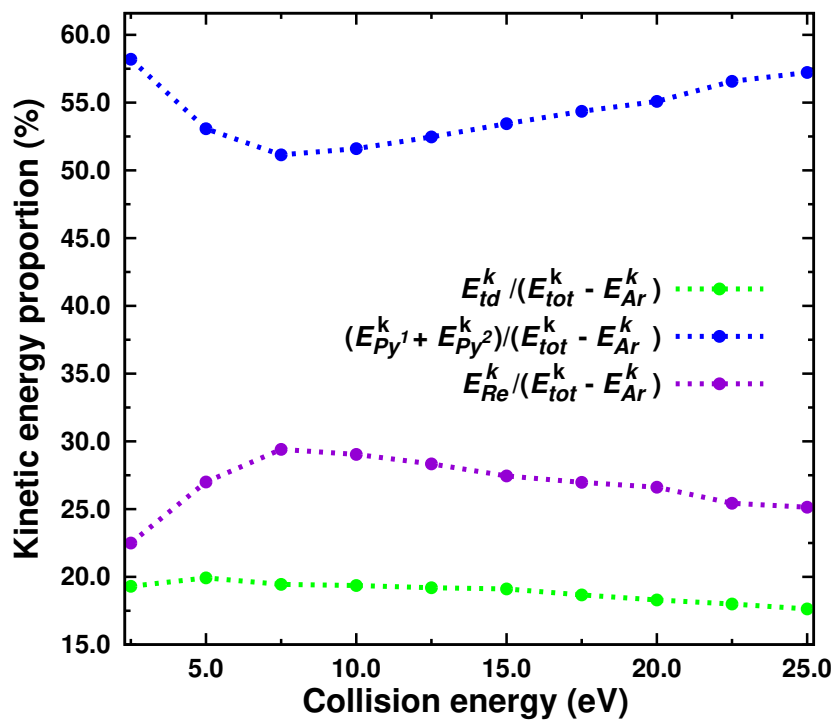

Fig. 7 Kinetic energy proportion after collision of $\mathrm{Py}_{2}^{+}$with Argon as a function of collision energy.

netic energy $\left(E_{t d}^{k}\right)$ remains almost constant (very slight decrease from about $20 \%$ to $18 \%$ of the dimer kinetic energy), this is not the case for the other two contributions. For collision energies below $7.5 \mathrm{eV}$, the proportion of the kinetic energy associated to the center of mass relative velocities increases whereas the opposite is observed for the monomers rovibrational kinetic energy. These two trends are reversed above $7.5 \mathrm{eV}$.

Again, it is convenient to analyse separately the kinetic energy partition for trajectories leading to dissociation or not as done in Figure 8 for $E_{t d}^{k}, E_{R e}^{k}$ and $E_{P y^{1}}^{k}+E_{P y^{2}}^{k}$. In the absence of dissociation, the transferred energy is either small or redistributed over all the vibrational modes of the dimer, leading to small values for $E_{R e-u d}^{k}$ (mean value always below $0.04 \mathrm{eV}$ ). The monomers rovibrational kinetic energies remain constant with an increase for collision energies above $20 \mathrm{eV}$, indicating that the slight increase of transferred 


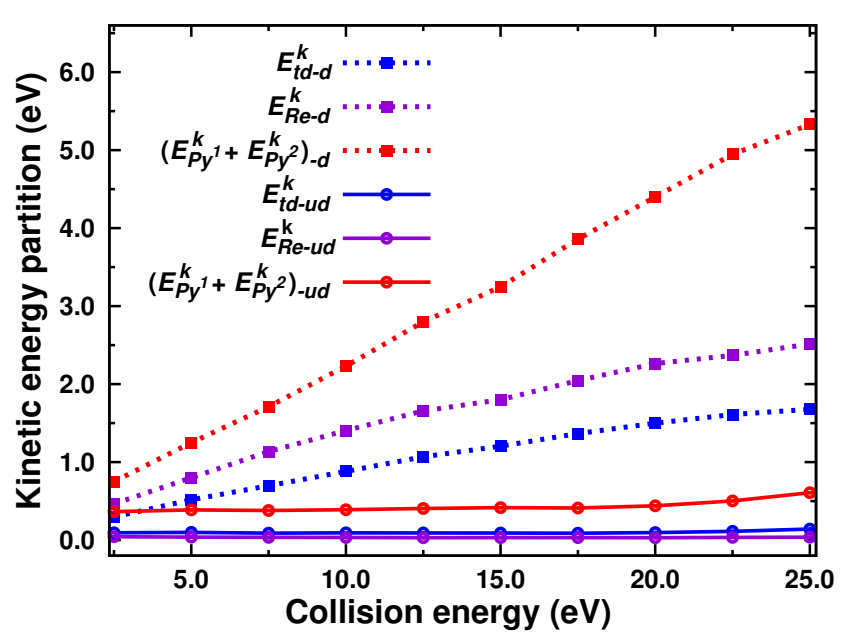

Fig. 8 Kinetic energy partition for dissociated (-d) and undissociated (-ud) trajectories at the end of the MD simulation as a function of collision energy.

energy results in a heating of the monomers, as already inferred from Figure 6. Once a dimer dissociates, the two pyrene molecules relative kinetic energy $E_{R e-d}^{k}$ cannot be transferred back to the intramolecular modes and its mean value is never negligible with respect to the monomers rovibrational kinetic energies $\left(E_{P y^{1}}^{k}+E_{P y^{2}}^{k}\right)_{-d}$. However, although the slope of $\left(E_{P y^{1}}^{k}+E_{P y^{2}}^{k}\right)_{-d}$ remains constant with collision energies, that of $E_{R e-d}^{k}$ clearly decreases. This is in line with the analysis of Figure 6 showing that the amount of energy transferred to the dissociative modes remains constant for high collision energies whereas the monomers are getting more internal energy.

Finally, we have computed some characteristic timescales which are presented in Figure 9. They correspond to the timescales for the Argon with its initial velocity to travel across some characteristic distances, namely, a C-H (1.10 $\AA$ ) or a C-C bond (1.40 $\AA$ ) and the largest molecular axis $(9.25 \AA)$. These timescales can be compared with those of the pyrene dimer vibrational modes as an efficient energy transfer would be favored by similar orders of magnitudes. The intermolecular dimer modes possibly mixed with very soft folding modes are lying within the $70-120 \mathrm{~cm}^{-1}$ spectral range [61] with corresponding half-periods of 130-240 fs. These timescales are of the same order of magnitude as the time for the Argon to travel across the largest pyrene axis for collision energies below $10 \mathrm{eV}$. Typical frequencies for intramolecular non-soft modes are lying from $500 \mathrm{~cm}^{-1}$ to $3000 \mathrm{~cm}^{-1}$ (C-H stretching modes), leading to half-periods of 5-33 fs. For all the simulated collision energies, the characteristic times required for the Argon to travel across typical $\mathrm{C}-\mathrm{H}$ or $\mathrm{C}-\mathrm{C}$ bond distances belong to the same order of magnitude as

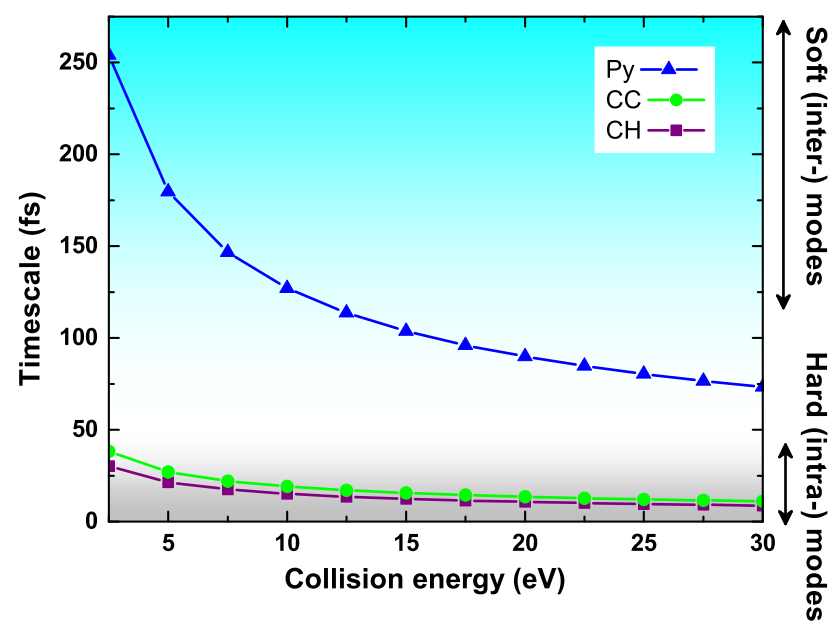

Fig. 9 Timescales, as a function of center of mass collision energy, for Argon to travel across some typical distances: a carbon-carbon bond (green), a carbon-hydrogen bond (purple) or the largest axis of the pyrene molecule (blue).

some of the intramolecular hard modes. Therefore, it appears from this qualitative description that the collision energy transfer toward the intermolecular modes is easier at collision energies lower than $10 \mathrm{eV}$ whereas the transfer toward intramolecular modes is efficient for all the simulated collision energies. This is actually in line with the fact that the part of the absorbed collision energy taken by the non-soft intramocular modes is increasing with the collision energy at the expense of that taken by the intermolecular and soft intramolecular modes, in agreement with the previous energy analysis (Figures 6, 7 and 8).

\subsubsection{Efficiency of energy transfer within the dimer}

In this section, we address how the energy is shared inside the dimer after the collision. In particular, we look at the efficiency of energy transfer between the intramolecular modes of each unit and the intermolecular modes. The amount of deposited energy as well as its partition between the intramolecular modes of each molecule and the intermolecular modes is strongly dependent on the collision condition: the impact parameter, the orientation of the dimer, whether a head on collision occurs with one of the dimer atoms (and its nature, carbon or hydrogen). This results in very different evolutions of the subsequent energy flows for which precise values concerning timescales can hardly be derived. Nevertheless, the analysis of the trajectories allows to identify some characteristic behaviors. In order to estimate the thermalization process efficiency, the instantaneous intra and intermolecular kinetic tem- 

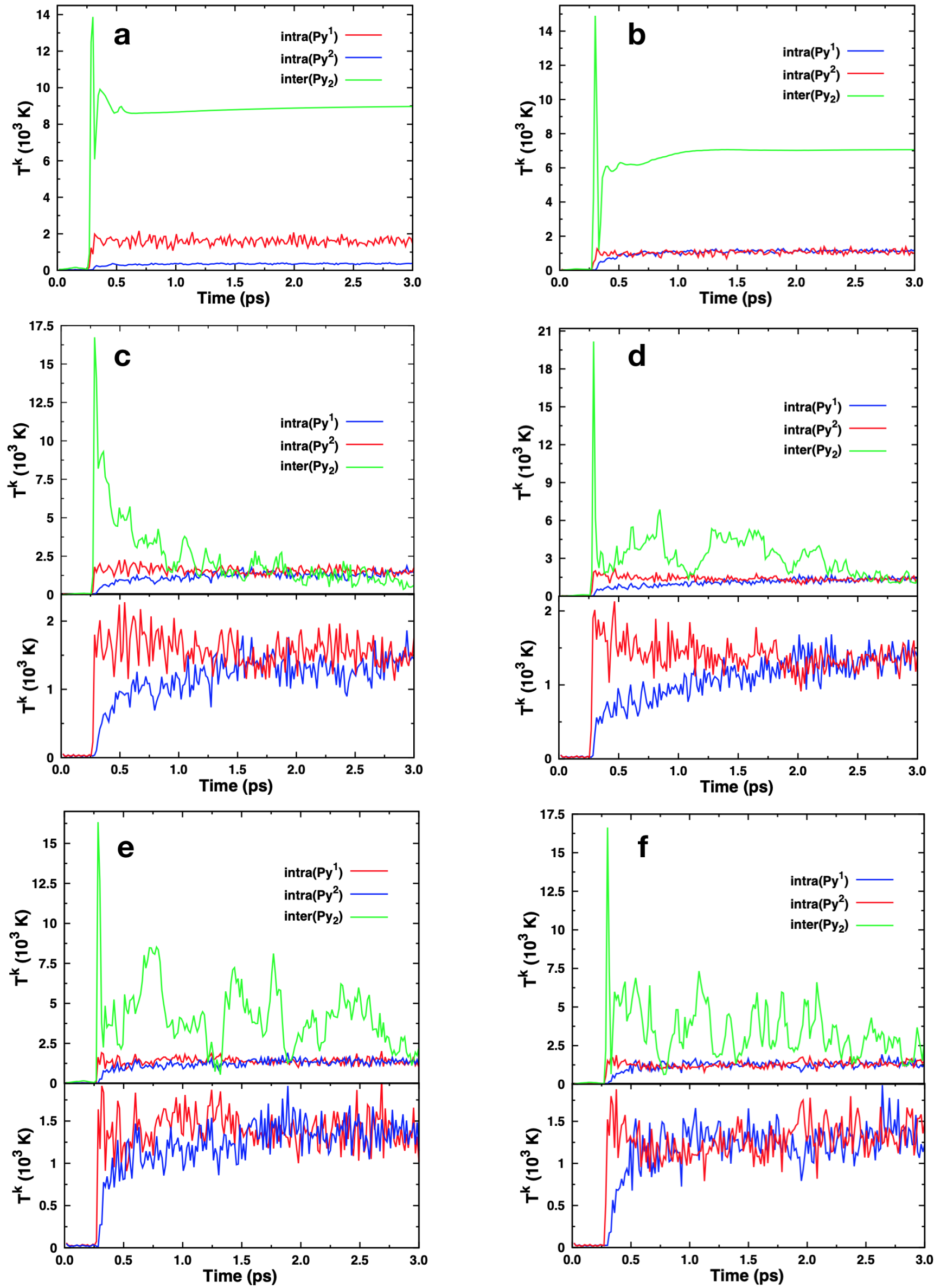

Fig. 10 Instantaneous kinetic temperatures as a function of time for intra and intermolecular modes of the pyrene dimer at a collision energy of $22.5 \mathrm{eV}$. Impact parameters $b$ are (a) 2, (b) 3, (c) 0, (d) 2.5, (e) 2, and (f) 2 A. In cases (a) and (b) dissociation takes place whereas in the other cases the dimer remains undissociated at the end of the simulation. In (c) to (f) the lower panel is a vertical zoom of the corresponding intramolecular parts in upper panel. 
peratures are evaluated using the formula:

$$
T^{k}=2 \frac{<E^{k}>}{n k_{b}}
$$

where $n$ is the number of involved modes and $E^{k}$ is the kinetic energy for the intra or intermolecular modes (see Eqs. 2). $T^{k}$ is plotted in Figure 10 for some selected trajectories obtained for collision energies of $22.5 \mathrm{eV}$ and various impact parameters. The evolution of the corresponding energies $\left(E_{\text {intra }}^{k}, E_{\text {intra }}^{k}\right.$ and $\left.E_{\text {inter }}^{k}\right)$ are presented in Figure 4 of the supplementary materials. In Figure 10, simulations (a) and (b) correspond to trajectories for which dissociation occurred, whereas the dimer remained intact in the other simulations. In the first simulation (a), a larger amount of energy is deposited in the first monomer with respect to the second one. The dissociation occurs before an efficient energy transfer takes place between the two monomers, leading to one hot monomer and one cold monomer at the end of the simulation. The situation is slightly different in the second dissociation trajectory (b): there is a much smaller difference between the energies received during the collission by each of the monomers. One can observe that the equilibration of the two monomers intramolecular energies can take place before dissociation, leaving the two monomers with similar energies/kinetic temperatures. In the other four pictures (c, d, e, and f), corresponding to undissociated trajectories, one can see that the thermalization between the two monomers intramolecular modes occurs with timescales from 0.2 to $1.5 \mathrm{ps}$. On the other side, the energy equilibration between intra and intermolecular modes takes more time. Indeed, the thermalization is almost achieved in simulations (c) and (d) at 1.5 and $2.5 \mathrm{ps}$, respectively, but would take more than the simulated duration 3 ps for trajectories (e) and (f). As a conclusion of these trajectories analyses, it seems that the thermalization between intramolecular modes of the two monomers is relatively efficient (on the order of ps). On the other hand, the thermalization with the intermolecular modes is less efficient and sometimes is not observed during the simulated time of $3 \mathrm{ps}$. The direct dissociation of the dimer is a fast process (on the order of a few tenths of ps) which may prevent the thermalization taking place, leading to monomer temperatures reflecting the initial energy deposition.

\section{Conclusions}

We carried out a QM/MM dynamics study of the collision of $\mathrm{Py}_{2}^{+}$with Argon at various collision energies. Argon was treated as a polarisable MM particle and $\mathrm{Py}_{2}^{+}$was treated using the SCC-DFTB method. The TOF mass spectra of parent $\mathrm{Py}_{2}^{+}$and dissociation product $\mathrm{Py}^{+}$were simulated by the PST using the MD outputs at a centre of mass collision energy of $17.5 \mathrm{eV}$. With respect to TOF mass spectra extracted from pure PST simulations, considering non-statistical dissociation processes that take place before the energy redistribution from MD simulations improves the match between experimental and theoretical TOF spectra. The agreement between the measured and simulated mass spectra peak shapes and positions shows that the essence of the collision induced dissociation is captured by the simulation. It appears that the TOF spectra mostly result from dimers dissociating on short timescales (during the MD simulation) and the remaining minor contribution is from dimers dissociating at longer timescales (the second step, during PST calculation). This indicates that $\mathrm{Py}_{2}^{+}$primarily engages a direct dissociation path after collision.

The extraction of snapshots from the MD simulations allows to visualize the collision processes. It shows that the evolution of the trajectories either toward a dissociation or a redistribution of the transferred energy strongly depends on the initial collision conditions. Intramolecular fragmentation of the monomers occurred only for collision energies above $25 \mathrm{eV}$. The dissociation cross sections show a steep increase for collision energies below $7.5 \mathrm{eV}$ and remain almost constant for collision energies greater than $10 \mathrm{eV}$. The dissociation cross section of $\mathrm{Py}_{2}^{+}$increases when dissociation occurring on longer timescale is included. As such, the dissociation cross section computed from the MD+PST model at the centre of mass collision energy of $17.5 \mathrm{eV}$ is slightly higher than the value derived from pure MD simulations. The analysis of the partition of the final kinetic energy as a function of the collision energy shows how the absorbed energy is shared between the dissociative modes and the heating of individual monomers. It shows that above $7.5 \mathrm{eV}$, increasing the collision energy mostly results in an increase of the intramolecular energy. The qualitative analysis of the different timescales involved in the collision further supports the kinetic energy partition analysis. Finally, the analysis of energy transfer efficiency within the dimer suggests that direct dissociation is too fast to allow significant thermalization of the system. On the other hand, when there is no dissociation, thermalization can occur with a faster equilibration between the intramolecular modes of the two units than with the intermolecular modes.

The present results can be compared with experimental and theoretical works discussing the direct and indirect fragmentation of PAH and PAH clusters submitted to higher energy collisions $[68,85]$. These au- 
thors showed that the nuclear stopping power dominates over the electronic one below $1 \mathrm{keV}$, giving a justification to our approach based on classical MD and PST. They also showed that the direct non-statistical $\mathrm{PAH}$ fragmentation (knock-out) is an efficient process above $20 \mathrm{eV}$. This is in line with the fact that monomer fragmentation was only observed in our MD simulations above $25 \mathrm{eV}$. Our work shows that, for PAH clusters, a regime exists below this collision energy where the dimer dissociation is governed by non-statistical processes.

In this present work, the collision process, dissociation path, energy partition and distribution, and the efficiency of energy transfer were deeply explored for the $\mathrm{Py}_{2}^{+}$system, which can provide valuable reference for the CID study of larger PAH cation clusters. Beyond the specific study of $\mathrm{Py}_{2}^{+}$dissociation, the methodology paves the way for future analysis of CID experiments like dissociation of molecular clusters such as waterUracil clusters.

Acknowledgements The authors acknowledge the supercomputing facility of CALMIP for generous allocation of computer resources. The authors declare that there has been no significant financial support for this work.

\section{References}

1. N.A. Eaves, S.B. Dworkin, M.J. Thomson, Proc. Comb. Inst. 35(2), 1787 (2015)

2. Q. Mao, A.C.T. van Duin, K.H. Luo, Carbon 121, 380 (2017)

3. C. Saggese, S. Ferrario, J. Camacho, A. Cuoci, A. Frassoldati, E. Ranzi, H. Wang, T. Faravelli, Combust. Flame 162(9), 3356 (2015)

4. D. Aubagnac-Karkar, A. El Bakali, P. Desgroux, Combust. Flame 189, 190 (2018)

5. T.S. Totton, D. Chakrabarti, A.J. Misquitta, M. Sander, D.J. Wales, M. Kraft, Combust. Flame 157(5), 909 (2010)

6. S.H. Chung, A. Violi, Proc. Combust. Inst. 33(1), 693 (2011)

7. C.S. Wang, N.C. Bartelt, R. Ragan, K. Thürmer, Carbon 129, 537 (2018)

8. H. Sabbah, L. Biennier, S.J. Klippenstein, I.R. Sims, B.R. Rowe, J. Phys. Chem. Lett. 1(19), 2962 (2010)

9. E.M. Adkins, J.A. Giaccai, J.H. Miller, Proc. Comb. Inst. 36(1), 957 (2017)

10. D. Chakraborty, H. Lischka, W.L. Hase, The Journal of Physical Chemistry A 124(43), 8907 (2020)

11. A. Léger, J.L. Puget, Astron. Astrophys. 137, L5 (1984)
12. L.J. Allamandola, A.G.G.M. Tielens, J.R. Barker, Astrophys. J. Lett. 290, L25 (1985)

13. M. Rapacioli, C. Joblin, P. Boissel, Astron. Astrophys. 429, 193 (2005)

14. O. Berné, C. Joblin, Y. Deville, J.D. Smith, M. Rapacioli, J.P. Bernard, J. Thomas, W. Reach, A. Abergel, Astron. Astrophys 469, 575 (2007)

15. O. Berné, C. Joblin, M. Rapacioli, J. Thomas, J.C. Cuillandre, Y. Deville, Astron. Astrophys 479, L41 (2008)

16. L. Lei, Y. Yao, J. Zhang, D. Tronrud, W. Kong, C. Zhang, L. Xue, L. Dontot, M. Rapacioli, J. Phys. Chem. Lett. 11(3), 724 (2020)

17. M. Goulart, M. Kuhn, B. Rasul, J. Postler, M. Gatchell, H. Zettergren, P. Scheier, O. Echt, Phys. Chem. Chem. Phys. 19, 27968 (2017)

18. Ö. Birer, E. Yurtsever, J. Mol. Struct. 1097, 29 (2015)

19. T. Beitz, R. Laudien, H.G. Löhmannsröben, B. Kallies, J. Phys. Chem. A 110(10), 3514 (2006)

20. C. Joblin, L. Dontot, G.A. Garcia, F. Spiegelman, M. Rapacioli, L. Nahon, P. Parneix, T. Pino, P. Bréchignac, J. Phys. Chem. Lett. 8(15), 3697 (2017)

21. J.E. Roser, A. Ricca, Astrophys. J. 801(2), 108 (2015)

22. A.K. Lemmens, S. Gruet, A.L. Steber, J. Antony, S. Grimme, M. Schnell, A.M. Rijs, Phys. Chem. Chem. Phys. 21, 3414 (2019)

23. S.E. Fioressi, R.C. Binning, D.E. Bacelo, Chem. Phys. Lett. 454(4), 269 (2008)

24. H. Friha, G. Féraud, T. Pino, P. Parneix, Z. Dhaouadi, P. Bréchignac, EAS Publ. Ser. 58, 373 (2012)

25. M. Schmidt, A. Masson, C. Bréchignac, Int. J. Mass Spectrom. 252(2), 173 (2006)

26. M. Gatchell, M.H. Stockett, N. de Ruette, T. Chen, L. Giacomozzi, R.F. Nascimento, M. Wolf, E.K. Anderson, R. Delaunay, V. Vizcaino, P. Rousseau, L. Adoui, B.A. Huber, H.T. Schmidt, H. Zettergren, H. Cederquist, Phys. Rev. A 92, 050702 (2015)

27. A.I.S. Holm, H. Zettergren, M. Gatchell, H.A.B. Johansson, F. Seitz, H.T. Schmidt, P. Rousseau, A. Ławicki, M. Capron, A. Domaracka, E. Lattouf, S. Maclot, R. Maisonny, J.Y. Chesnel, B. Manil, L. Adoui, B.A. Huber, H. Cederquist, J. Phys. Conf. Ser. 388(1), 012051 (2012)

28. H.A.B. Johansson, H. Zettergren, A.I.S. Holm, F. Seitz, H.T. Schmidt, P. Rousseau, A. Lawicki, M. Capron, A. Domaracka, E. Lattouf, S. Maclot, R. Maisonny, B. Manil, J.Y. Chesnel, L. Adoui, B.A. Huber, H. Cederquist, Phys. Rev. A 84(4), 
$043201(2011)$

29. A.I.S. Holm, H. Zettergren, H.A.B. Johansson, F. Seitz, S. Rosén, H.T. Schmidt, A. Ławicki, J. Rangama, P. Rousseau, M. Capron, R. Maisonny, L. Adoui, A. Méry, B. Manil, B.A. Huber, H. Cederquist, Phys. Rev. Lett. 105(21), 213401 (2010)

30. M. Gatchell, P. Rousseau, A. Domaracka, M.H. Stockett, T. Chen, H.T. Schmidt, J.Y. Chesnel, A. Méry, S. Maclot, L. Adoui, B.A. Huber, H. Zettergren, H. Cederquist, Phys. Rev. A 90, 022713 (2014)

31. R. Delaunay, M. Gatchell, P. Rousseau, A. Domaracka, S. Maclot, Y. Wang, M.H. Stockett, T. Chen, L. Adoui, M. Alcamí, F. Martín, H. Zettergren, H. Cederquist, B.A. Huber, J. Phys. Chem. Lett. 6(9), 1536 (2015)

32. S. Zamith, M.C. Ji, J.M. L'Hermite, C. Joblin, L. Dontot, M. Rapacioli, F. Spiegelman, J. Chem. Phys. 151(19), 194303 (2019)

33. F. Gámez, A.R. Hortal, B. Martínez-Haya, J. Soltwisch, K. Dreisewerd, J. Mass Spectrom. 49(11), 1127 (2014)

34. J. Zhen, T. Chen, A.G.G.M. Tielens, Astrophys. J. 863(2), 128 (2018)

35. P. Bréchignac, M. Schmidt, A. Masson, T. Pino, P. Parneix, C. Bréchignac, Astron. Astrophys. 442(1), 239 (2005)

36. T. Chen, Astrophys. J. 866(2), 113 (2018)

37. E. Yurtsever, Theo. Chem. Acc. 127(3

38. M. Piacenza, S. Grimme, J. Am. Chem. Soc. $\mathbf{1 2 7}(42), 14841$ (2005)

39. R. Podeszwa, J. Chem. Phys. 132, 044704 (2010)

40. Y. Zhao, D.G. Truhlar, J. Phys. Chem. C 112, 4061 (2008)

41. J.C. Sancho-García, A.J. Pérez-Jiménez, Phys. Chem. Chem. Phys. 11, 2741 (2009)

42. M. Rapacioli, F. Spiegelman, D. Talbi, T. Mineva, A. Goursot, T. Heine, G. Seifert, J. Chem. Phys. 130, 244304 (2009)

43. O.I. Obolensky, V.V. Semenikhina, A.V. Solov'yov, W. Greiner, Int. J. Quant. Chem. 107(6), 1335 (2007)

44. M. Bartolomei, F. Pirani, J.M.C. Marques, J. Phys. Chem. C 121(26), 14330 (2017)

45. A. Ricca, J. Charles W. Bauschlicher, L.J. Allamandola, Astrophys. J. 776(1), 31 (2013)

46. F. Piuzzi, I. Dimicoli, M. Mons, P. Millié, V. Brenner, Q. Zhao, B. Soep, A. Tramer, Chem. Phys. $\mathbf{2 7 5}(1), 123$ (2002)

47. T.S. Totton, A.J. Misquitta, M. Kraft, Chem. Phys. Lett. 510(1), 154 (2011)

48. P. Grančič, R. Bylsma, H. Meekes, H.M. Cuppen, Cryst. Growth Des 15(4), 1625 (2015)
49. J.D. Herdman, J.H. Miller, J. Phys. Chem. A $\mathbf{1 1 2}(28), 6249$ (2008)

50. D.C. Easter, J.A. Roof, L.J. Butts, J. Phys. Chem. A 111(50), 12914 (2007)

51. T.S. Totton, A.J. Misquitta, M. Kraft, Phys. Chem. Chem. Phys. 14, 4081 (2012)

52. K. Bowal, J.W. Martin, M. Kraft, Carbon 143, 247 (2019)

53. L. Pascazio, M. Sirignano, A. D'Anna, Combust. Flame 185, 53 (2017)

54. D. Chen, T.S. Totton, J.W.J. Akroyd, S. Mosbach, M. Kraft, Carbon 67, 79 (2014)

55. M. Nakamura, A. Ichimura, Physica Scripta 2013(T156), 014063 (2013)

56. H. Takeuchi, Comput. Theor. Chem. 1021, 84 (2013)

57. F. Calvo, E. Yurtsever, Ö. Birer, J Phys Chem A 120(10), 1727 (2016)

58. J. Gräfenstein, D. Cremer, Theo. Chem. Acc. : Theo. Comp. Model. 123, 171 (2009)

59. L. Dontot, F. Spiegelman, M. Rapacioli, J. Phys. Chem. A 123(44), 9531 (2019)

60. L. Dontot, N. Suaud, M. Rapacioli, F. Spiegelman, Phys. Chem. Chem. Phys. 18, 3545 (2016)

61. L. Dontot, F. Spiegelman, S. Zamith, M. Rapacioli, Eur. Phys. J. D 74(11), 1 (2020)

62. D. Porezag, T. Frauenheim, T. Köhler, G. Seifert, R. Kaschner, Phys. Rev. B 51, 12947 (1995)

63. G. Seifert, D. Porezag, T. Frauenheim, Int. J. Quantum Chem. 58, 185 (1996)

64. M. Elstner, D. Porezag, G. Jungnickel, J. Elsner, M. Haugk, T. Frauenheim, S. Suhai, G. Seifert, Phys. Rev. B 58, 7260 (1998)

65. F. Spiegelman, N. Tarrat, J. Cuny, L. Dontot, E. Posenitskiy, C. Martí, A. Simon, M. Rapacioli, Adv. Phys. X 5(1), 1710252 (2020)

66. M. Rapacioli, F. Spiegelman, A. Scemama, A. Mirtschink, J. Chem. Theor. Comput. 7, 44 (2011)

67. M. Gatchell, H. Zettergren, H. Cederquist, H. Schmidt, Molecular hole punching : Impulse driven reactions in molecules and molecular clusters. Ph.D. thesis, Stockholm University, Stockholm (2016)

68. M. Gatchell, H. Zettergren, J. Phys. B 49(16), $162001(2016)$

69. S. Zamith, J.M. L'Hermite, L. Dontot, L. Zheng, M. Rapacioli, F. Spiegelman, C. Joblin, J. Chem. Phys. 153, 054311 (2020)

70. M. Elstner, G. Seifert, Philos. Trans. R. Soc. A 372(2011), 20120483 (2014)

71. F. Spiegelman, N. Tarrat, J. Cuny, L. Dontot, E. Posenitskiy, C. Martí, A. Simon, M. Rapacioli, 
Adv Phys-X 5(1), 1710252 (2020)

72. C. Iftner, A. Simon, K. Korchagina, M. Rapacioli, F. Spiegelman, J. Chem. Phys. 140(3), 034301 (2014)

73. J. Frenzel, A.F. Oliveira, N. Jardillier, T. Heine, G. Seifert, Semi-relativistic, self-consistent charge Slater-Koster tables for density-functional based tight-binding (DFTB) for materials science simulations (TU Dresden, 2004-2009)

74. J. Li, T. Zhu, C. Cramer, D. Truhlar, J. Phys. Chem. A 102, 1820 (1998)

75. E. Kukk, D. Ha, Y. Wang, D.G. Piekarski, S. DiazTendero, K. Kooser, E. Itälä, H. Levola, M. Alcamí, E. Rachlew, et al., Phys. Rev. A 91(4), 043417 (2015)

76. A. Simon, M. Rapacioli, G. Rouaut, G. Trinquier, F. Gadéa, Philos. Trans. R. Soc. A 375(2092), 20160195 (2017)

77. A. Simon, J.P. Champeaux, M. Rapacioli, P. Moretto-Capelle, X. Gadéa, Florent, M. Sence, Theor. Chem. Acc. 137(7), 106 (2018)

78. M. Rapacioli, S. Cazaux, N. Foley, A. Simon, R. Hoekstra, T. Schlathölter, Phys. Chem. Chem. Phys. 20(35), 22427 (2018)

79. S. Nosé, J. Chem. Phys. 81(1), 511 (1984)

80. W.G. Hoover, Phys. Rev. A 31(3), 1695 (1985)

81. T. Heine, M. Rapacioli, S. Patchkovskii, J. Frenzel, A. Koster, P. Calaminici, H. Duarte, S. Escalante, R. Flores-Moreno, A. Goursot. demonnano (2009)

82. I. Braud, S. Zamith, J.M. L'Hermite, Rev. Sci. Instrum. 88(4), 043102 (2017)

83. F. Chirot, S. Zamith, P. Labastie, J.M. L'Hermite, Rev. Sci. Instrum. 77(6), 063108 (2006)

84. D. Levine, R.B. Bernstein, Molecular Reaction Dynamics and Chemical Reactivity (Oxford University Press, 1987)

85. T. Chen, M. Gatchell, M.H. Stockett, J.D. Alexander, Y. Zhang, P. Rousseau, A. Domaracka, S. Maclot, R. Delaunay, L. Adoui, et al., J. Chem. Phys. 140(22), 224306 (2014) 